\title{
Raman spectroscopy of ferromagnetic $\mathrm{CrO}_{2}$
}

\author{
M. N. Iliev, A. P. Litvinchuk, H.-G. Lee, and C. W. Chu \\ Texas Center for Superconductivity, University of Houston, Houston, Texas 77204-5932
}

\author{
A. Barry and J. M. D. Coey \\ Department of Physics, Trinity College, Dublin 2, Ireland
}

(Received 11 January 1999)

\begin{abstract}
Polarized Raman spectra of the ferromagnetic metal $\mathrm{CrO}_{2}$ were measured in a broad temperature range including $T_{c} \approx 390 \mathrm{~K}$. The Raman-allowed modes of $A_{1 g}, B_{1 g}, B_{2 g}$, and $E_{g}$ symmetry were identified and compared to the corresponding modes of isostructural $\mathrm{TiO}_{2}$. The two low-frequency Raman lines are relatively narrow, indicating weak scattering of the corresponding $B_{1 g}$ and $E_{g}$ phonons. Whereas the Raman intensities are not visibly affected by the magnetic ordering, the dependences on temperature of the phonon wave numbers exhibit peculiarities that could be attributed to a spin-phonon interaction. The variations with $T$ of the phonon linewidths follow at low temperatures the dependence expected for phonon-phonon scattering. An additional broadening at higher temperatures with a maximum near $T_{c}$ is tentatively assigned to scattering from collective spin fluctuations. [S0163-1829(99)00825-5]
\end{abstract}

Chromium dioxide, $\mathrm{CrO}_{2}$, has attracted significant interest as it has a unique electronic band structure, resulting in halfmetallic ferromagnetism $\left(T_{c} \approx 390 \mathrm{~K}\right)$ with completely spinpolarized electrons at $E_{F} \cdot{ }^{1-4}$ Although there have been numerous reports on the magnetic, electric, and structural properties of $\mathrm{CrO}_{2}$, there are few data on its phonon spectra. Symmetry analysis of the zone-center phonons of $\mathrm{CrO}_{2}$ (space group $\mathrm{P}_{2} / \mathrm{mnm}$ ) reveals that there are four Ramanactive phonon modes of $A_{1 g}, B_{1 g}, B_{2 g}$, and $E_{g}$ symmetry and four infrared-active phonon modes $\left(A_{2 u}+3 E_{u}\right){ }^{6}$ The atomic motions corresponding to the Raman modes are shown in Fig. 1. Lewis et al. ${ }^{2}$ predicted the frequency of the $A_{1 \mathrm{~g}}$ mode to be $587 \mathrm{~cm}^{-1}$. Basov et al. ${ }^{7}$ measured the optical conductivity in the infrared frequency range and found three peaks at 357,474 , and $573 \mathrm{~cm}^{-1}$, which should correspond to transverse optical phonons. No conclusion was drawn regarding the symmetry of these infrared-active vibrations.

In this work we report the polarized Raman spectra of $\mathrm{CrO}_{2}$ as obtained from (110)- and (001)-oriented thin films on $\mathrm{TiO}_{2}$ substrates of the same orientation. The Raman lines corresponding to the Raman-allowed phonon modes were unambiguously identified and their variation with temperature was studied between 5 and $420 \mathrm{~K}$.

In the upper part of Fig. 2 are shown the Raman spectra of $\mathrm{CrO}_{2}$ as obtained at $300 \mathrm{~K}$ with $632.8 \mathrm{~nm}$ laser excitation in four different scattering configurations, ${ }^{5}$ namely, $y^{\prime}\left(x^{\prime} x^{\prime}\right) \bar{y}^{\prime}$ $\left(A_{1 g}+B_{2 g}\right), y^{\prime}(z z) \bar{y}^{\prime}\left(A_{1 g}\right), y^{\prime}\left(z x^{\prime}\right) \bar{y}^{\prime}\left(E_{g}\right)$, and $z\left(x^{\prime} y^{\prime}\right) \bar{z}$ $\left(B_{1 g}\right)$ scattering configurations $\left(x^{\prime}, y^{\prime}\right.$, and $z$ correspond to the crystallographic directions $[1 \overline{1} 0],[110]$, and [001], respectively; in the brackets are the allowed phonon mode symmetries). In the lower part of Fig. 2 the corresponding spectra for the isostructural compound $\mathrm{TiO}_{2}$ are shown. The latter spectra are identical to those reported in the pioneering work of Porto et al. ${ }^{6}$ Figure 3 shows the temperature evolution of the spectra of $\mathrm{CrO}_{2}$ between 5 and $420 \mathrm{~K}$. These spectra were obtained with $514.5 \mathrm{~nm}$ excitation from the (110)-oriented film with incident polarization along $x^{\prime}$ and without an analyzer. Therefore they are a superposition of $y^{\prime}\left(x^{\prime} x^{\prime}\right) \bar{y}^{\prime} \quad\left(A_{1 g}+B_{2 g}\right)$ and $y^{\prime}\left(x^{\prime} z\right) \bar{y}^{\prime} \quad\left(E_{g}\right)$ spectra. In Table I are compared the wave numbers and linewidths of the Raman phonon lines of $\mathrm{CrO}_{2}$ and $\mathrm{TiO}_{2}$ at $300 \mathrm{~K}$ and $5 \mathrm{~K}$ as well as their relative intensities with $632.8 \mathrm{~nm}$ and 514.5 $\mathrm{nm}$ excitation. To calculate the relative intensities we fitted each phonon line by a Lorentzian and normalized its area to that of the $A_{1 g}$ line in the $x^{\prime} x^{\prime}$ spectrum with the same excitation and at the same temperature.

It follows from Figs. 2 and 3 and Table I that the Raman spectra of $\mathrm{CrO}_{2}$ and $\mathrm{TiO}_{2}$ exhibit definite differences, namely, (i) only the one-phonon lines are pronounced in the spectra of $\mathrm{CrO}_{2}$ in contrast to $\mathrm{TiO}_{2}$, where the intensity of two-phonon bands is comparable to that of the one-phonon lines; (ii) the $A_{1 g}$ and $B_{2 g}$ lines are of comparable intensity in $\mathrm{CrO}_{2}$, whereas in $\mathrm{TiO}_{2}$ the $B_{2 g}$ line is weaker by two orders of magnitude; and (iii) the Raman line corresponding to the $A_{1 g}$ mode is of comparable intensity with $x x\left(x^{\prime} x^{\prime}\right)$ and $z z$ scattering configurations for $\mathrm{CrO}_{2}$, whereas the $z z$

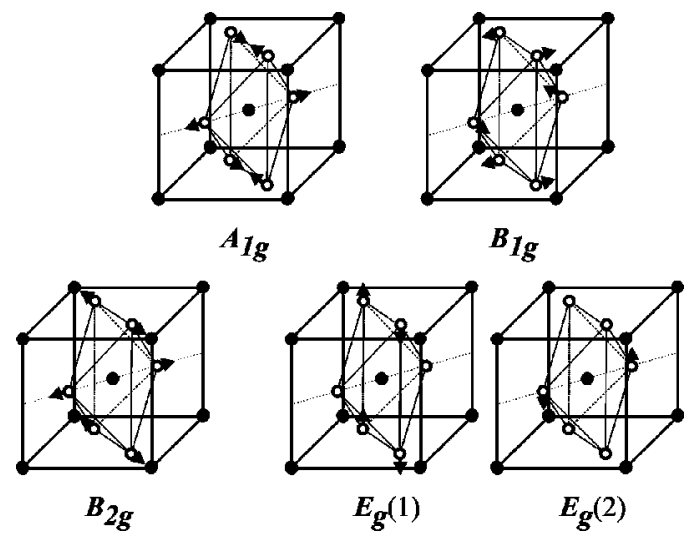

FIG. 1. Main atomic motions in the Raman modes of $\mathrm{CrO}_{2}$ (space group $\mathrm{P}_{2} / \mathrm{mnm}$ ). Only oxygen atoms participate in these modes. 


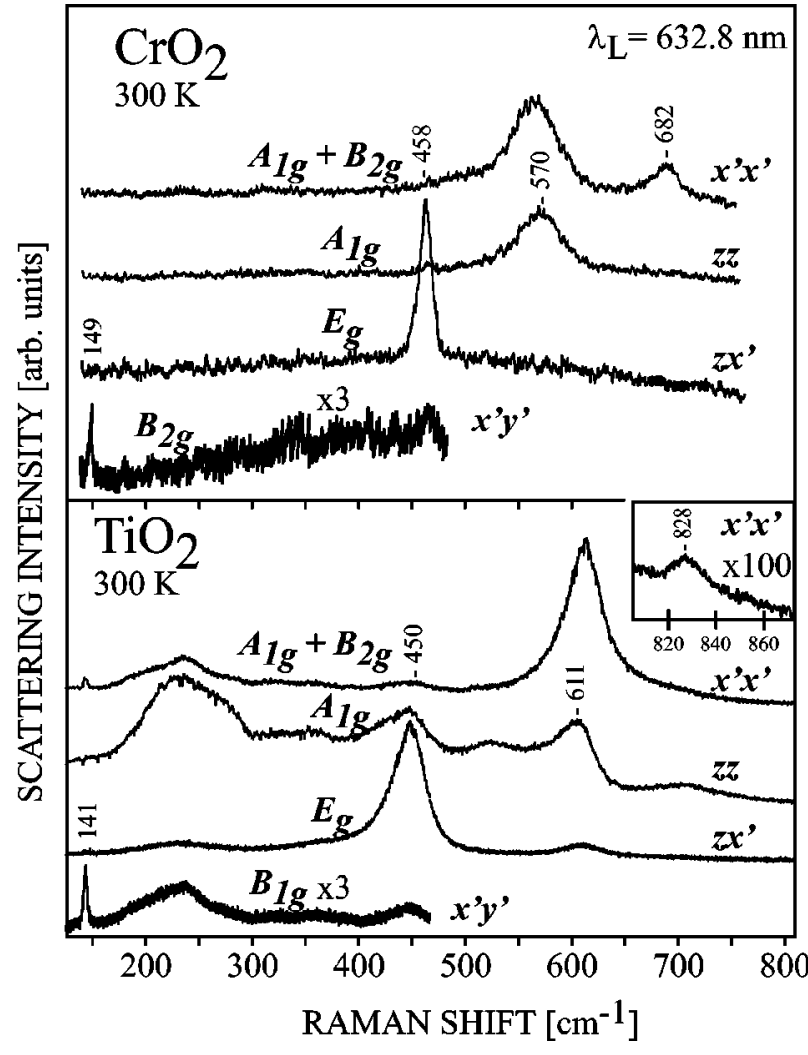

FIG. 2. Polarized Raman spectra of $\mathrm{CrO}_{2}$ and $\mathrm{TiO}_{2}$ as obtained at $300 \mathrm{~K}$ with $632.8 \mathrm{~nm}$ excitation.

component is much weaker in the case of $\mathrm{TiO}_{2}$.

It is plausible to assume that the above differences in the otherwise isostructural compounds are due mainly to the different intermediate electronic states in the Raman scattering process. $\mathrm{TiO}_{2}$ is a transparent material and the laser excitation energies (1.96 and $2.41 \mathrm{eV})$ used in our experiments are well below the gap for electronic transitions. In contrast, upon photon excitation the final states of both spin-up and spin-down electrons in $\mathrm{CrO}_{2}$ are in the corresponding conduction bands. ${ }^{3,8}$ The Raman-scattering efficiency $\eta$ for a given phonon can be expressed as ${ }^{9}$

$$
\eta=\left(\frac{\omega_{s}}{c}\right)^{4} L V\left|\mathbf{e}_{i} \cdot \mathcal{R} \cdot \mathbf{e}_{s}\right|^{2},
$$

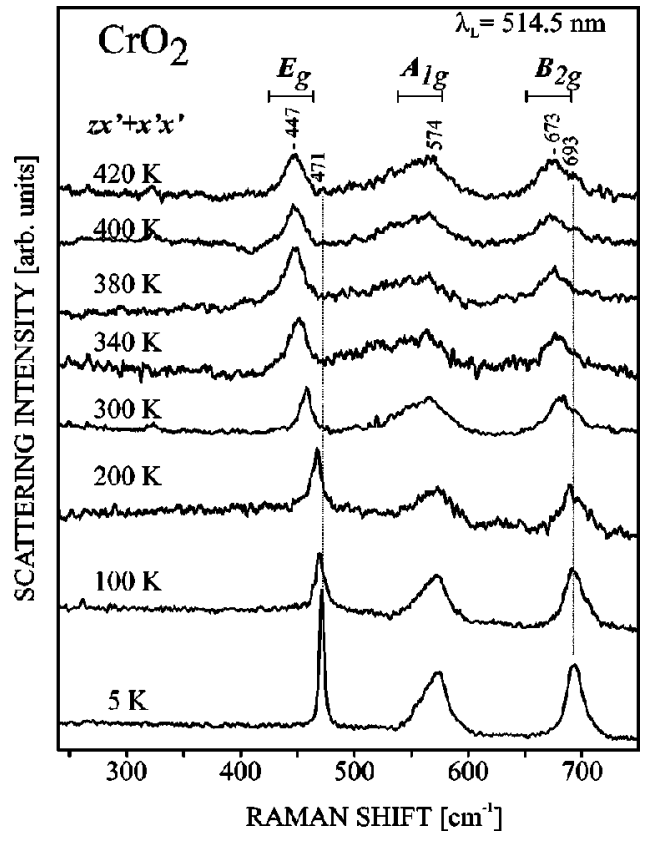

FIG. 3. Variations of the $x^{\prime} x^{\prime}+x^{\prime} z$ Raman spectrum of $\mathrm{CrO}_{2}$ between 5 and $420 \mathrm{~K}$. The excitation photon energy was $2.41 \mathrm{eV}$ $(514.5 \mathrm{~nm})$

where $i$ and $s$ are indices for incident and scattered radiation, $L$ is the scattering length defined for an absorbing material as $\left(\alpha_{i}+\alpha_{s}\right)^{-1}$ ( $\alpha$ is the absorption coefficient), $V$ is the volume of the sample producing the scattered radiation (for a parallel beam with area $A, V=L A), \mathbf{e}_{i}$ and $\mathbf{e}_{s}$ denote the incident and scattered polarization, and $\mathcal{R}$ is the corresponding Raman tensor. For the transparent range the second-rank Raman tensor $\mathcal{R}$ is defined as

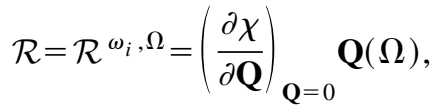

where $\mathbf{Q}(\mathbf{r}, t)=\mathbf{Q}(\mathbf{q}, \Omega) \cos (\mathbf{q} \cdot \mathbf{r}-\Omega t)$ are the atomic displacements associated with a phonon with wave vector $\mathbf{q}$ and frequency $\Omega$ and $\chi\left(\mathbf{k}_{i}, \omega_{i}, \mathbf{Q}\right)=\chi\left(\mathbf{k}_{i}, \omega_{i}\right)$ $+(\partial \chi / \partial \mathbf{Q})_{0} \mathbf{Q}(\mathbf{r}, t)+\cdots$ is the electric susceptibility. For photon energies within the range of direct interband elec-

TABLE I. Wave number and halfwidth of the Raman phonon lines of $\mathrm{CrO}_{2}$ and $\mathrm{TiO}_{2}$ at $300 \mathrm{~K}$ and $5 \mathrm{~K}$, and their relative intensities with $632.3 \mathrm{~nm}$ and $514.5 \mathrm{~nm}$ laser line excitation.

\begin{tabular}{lrccccccc}
\hline \hline $\begin{array}{l}\text { Raman } \\
\text { mode }\end{array}$ & $\begin{array}{c}T \\
(\mathrm{~K})\end{array}$ & $\begin{array}{c}\text { Position } \\
\left(\mathrm{cm}^{-1}\right)\end{array}$ & $\begin{array}{c}\text { Width } \\
\left(\mathrm{cm}^{-1}\right)\end{array}$ & $\begin{array}{c}\mathrm{CrO}_{2} \\
(632.8 \mathrm{~nm})\end{array}$ & $\begin{array}{c}\text { Intensity } \\
(541.5 \mathrm{~nm})\end{array}$ & $\begin{array}{c}\text { Position } \\
\left(\mathrm{cm}^{-1}\right)\end{array}$ & $\begin{array}{c}\mathrm{TiO}_{2} \\
\left(\mathrm{~cm}^{-1}\right)\end{array}$ & $\begin{array}{c}\text { Intensity } \\
(632.8 \mathrm{~nm})\end{array}$ \\
\hline$A_{1 g}$ & 5 & 574 & 24 & & $1.00(x x)$ & 612 & 21 & \\
& & & & & $1.61(z z)$ & & & \\
& 300 & 570 & 49 & $1.00(x x)$ & $1.00(x x)$ & 611 & 44 & $1.00(x x)$ \\
& & & & $0.59(z z)$ & $0.58(z z)$ & & & $0.33(z z)$ \\
$B_{1 g}$ & 5 & & & & & 144 & 3.5 & \\
& 300 & 149 & 3.0 & 0.01 & & 141 & 3.7 & 0.006 \\
$B_{2 g}$ & 5 & 693 & 16 & & 0.89 & & & \\
& 300 & 682 & 29 & 0.19 & 0.57 & 828 & 14.1 & 0.002 \\
$E_{g}$ & 5 & 471 & 4.5 & & & 454 & 10 & \\
& 300 & 458 & 11.5 & 0.22 & & 450 & 36 & 0.81 \\
\hline \hline
\end{tabular}


tronic transitions, in particular near a critical point, the nonzero components of $\mathcal{R}$ may significantly increase due to a strong modulation of combined electronic densities of states [and hence of $\chi\left(\omega_{i}\right)$ ] by particular atomic vibrations. Despite the strong increase of $\mathcal{R}$ in such resonance conditions, however, the scattering efficiency $\eta$ of a light-absorbing medium as a whole decreases due to the much stronger effect of the reduced scattering length $L$ and scattering volume $V$. The resonance conditions for $\mathrm{CrO}_{2}$ do change with variation of the excitation photon energy as manifested by the significant difference of the relative intensity of the $B_{2 g}$ mode with 632.8 and $514.5 \mathrm{~nm}$ excitation.

The resonant conditions (and hence of the relative Raman line intensities) are expected to change also for a fixed excitation photon energy provided the electronic structure undergoes changes. For ferromagnetic materials such changes could be induced by the spin ordering, resulting in a splitting of electronic bands into spin-polarized subbands. Such a magnetic-order-dependent Raman scattering has been reported, e.g., for the ferromagnetic semiconductors $\mathrm{CdCr}_{2} \mathrm{Se}_{4}$ and $\mathrm{CdCr}_{2} \mathrm{~S}_{4}$ where some of the Raman lines increase strongly upon cooling below the corresponding Curie temperatures. ${ }^{10}$ It has been shown that such a temperature behavior which is sensitive to the laser photon energy could be explained by the exchange splitting of the electronic bands resulting in a change of combined electronic densities of states near the energy of the laser excitation. ${ }^{11}$

It follows from Fig. 3, within the accuracy of our experiments, that no redistribution of the relative intensities of the Raman lines of $\mathrm{CrO}_{2}$ has been detected near $T_{c} \approx 390 \mathrm{~K}$. This is an indication that the combined electronic density of states near $2.4 \mathrm{eV}$ is not strongly affected by magnetic ordering. Such a result indicates that Hund's rule exchange splitting is still present up to the highest temperature in our experiments $(420 \mathrm{~K})$ and, therefore, the electronic structure of magnetically ordered domains above $T_{c}$ is very similar to the one in the ferromagnetic phase below $T_{c}$.

The variations with temperature of the position the $E_{g}$, $A_{1 g}$, and $B_{2 g}$ Raman lines are shown in the right panels of Fig. 4. While with temperature increasing from 5 to $420 \mathrm{~K}$ the $E_{g}$ and $B_{2 g}$ modes soften by $5.5 \%$ and $3 \%$, respectively, the softening of the $A_{1 g}$ mode is less than $1 \%$. As the experimental points are restricted to temperatures close to above $T_{c}$, no definite conclusion can be drawn about the effect of magnetic ordering on the phonon frequencies. Nevertheless, there are indications for anomalies near $T_{c}$ in the position of both the $A_{1 g}$ and $E_{g}$ modes.

The dependences of the phonon linewidth vs temperature, $\Gamma(T)$, are illustrated in the left panels of Fig. 4. At low temperatures the linewidth of the $E_{g}$ phonon is relatively low $\left(4.5 \mathrm{~cm}^{-1}\right)$. For the $B_{1 g}$ phonon, which was not measured at low temperatures, ${ }^{12}$ an even smaller linewidth is expected as at $300 \mathrm{~K}$ its width is only $3 \mathrm{~cm}^{-1}$, equal to the resolution of our spectrometer. The linewidth of a phonon line is a measure of the phonon scattering. The small widths of the low-frequency $B_{1 g}$ and $E_{g}$ phonons, therefore, provide an evidence for a weak scattering. In contrast, the much higher linewidths of the $A_{1 g}$ and $B_{2 g}$ phonons $\left(24 \mathrm{~cm}^{-1}\right.$ and $16 \mathrm{~cm}^{-1}$, respectively, at $5 \mathrm{~K}$ ) indicate that these phonons scatter much more strongly. This observation is consistent

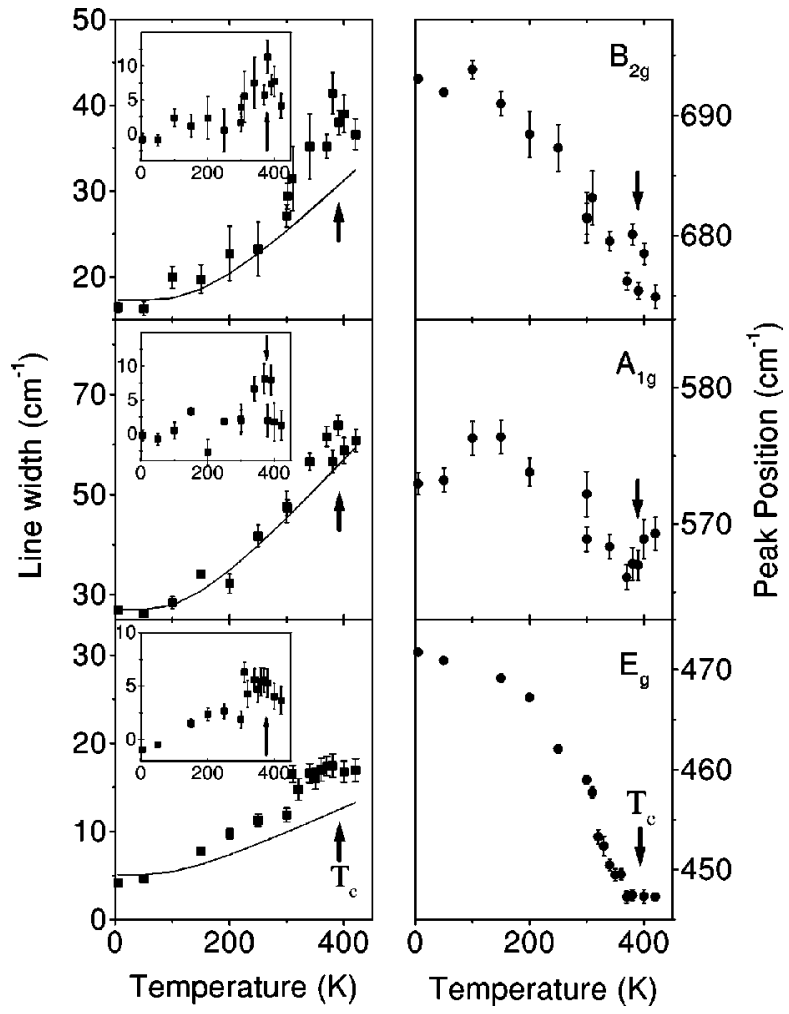

FIG. 4. Variations with temperature of the peak positions and linewidths of the $E_{g}, A_{1 g}$, and $B_{2 g}$ Raman lines of $\mathrm{CrO}_{2}$. The solid lines in the left panels represent the corresponding functions $\Gamma_{p p}$ which describe the phonon-phonon interaction. The insets show the additional broadening $\Delta \Gamma=\Gamma_{\exp }-\Gamma_{p p}$ assigned to magnetic scattering.

with the results of Basov et $a l^{7}$ who found that at low temperatures the electronic scattering below $500 \mathrm{~cm}^{-1}$ becomes negligible.

It is plausible to assume that the dependence $\Gamma(T)$ is governed by mainly phonon-phonon scattering (anharmonic decay), described by

$$
\Gamma_{p p}(\mathrm{~T})=\Gamma_{0}\left[1+\frac{2}{\exp \left(\hbar \Omega / 2 k_{B} \mathrm{~T}\right)-1}\right]
$$

where $\Gamma_{0}$ is the linewidth at $T=0$ and $\Omega$ is the phonon frequency. The function $\Gamma_{p p}(T)$, shown by solid curve in the left panels of Fig. 4, fits satisfactorily the experimental points at low temperatures. At high temperatures, however, the experimental points lie above $\Gamma_{p p}(T)$, indicating that another channel could also be involved in the scattering process. For a magnetic material like $\mathrm{CrO}_{2}$ such an additional channel could arise from scattering from the collective spin fluctuations (spin disorder scattering) that exist near $T_{c}$. If so, the contribution of spin-disorder scattering to the phonon linewidth should pass over a maximum near $T_{c}$. The differences $\Delta \Gamma=\Gamma_{\exp }-\Gamma_{p p}$, shown in the insets of Fig. 4, do have a maximum near $T_{c}$, although a more definite conclusion could be drawn only after extending the measurements to higher temperatures. It is worth noting here that the local 
maximum near $T_{c}$ of the phonon linewidth has recently been reported for another ferromagnetic compound, $\mathrm{SrRuO}_{3}$, which is also a "bad" metal. ${ }^{13}$

In conclusion, we measured the polarized Raman spectra of ferromagnetic metal $\mathrm{CrO}_{2}$ in a broad temperature range including $T_{c}$ and identified all four allowed Raman modes. The low-frequency Raman lines of $B_{1 g}$ and $E_{g}$ symmetry are very narrow, indicating weak phonon scattering, in particular at low temperatures. The Raman intensities are not strongly affected by the magnetic ordering, whereas the line position vs $T$ dependences change their slope near $T_{c}$ for the $A_{1 g}$ and $E_{g}$ modes. At low temperatures the variations with $T$ of the phonon linewidths follow the dependence expected for a phonon-phonon scattering. The additional broadening which appears with approaching $T_{c}$ is tentatively assigned to the scattering of phonons from collective spin fluctuations.

This work was supported by ARPA MDA 972-90-J-1001, the State of Texas through the Texas Center of Superconductivity, and in part by the MRSEC Program of the National Science Foundation under Grant No. DMR-9632667.
${ }^{1}$ K. Schwarz, J. Phys. F 16, L211 (1986).

${ }^{2}$ S.P. Lewis, P.B. Allen, and T. Sasaki, Phys. Rev. B 55, 10253 (1997).

${ }^{3}$ M.A. Korotin, V.I. Anisimov, D.I. Khomskii, and G.A. Sawatzky, Phys. Rev. Lett. 80, 4305 (1998).

${ }^{4}$ R.J. Soulen, Jr., J.M. Byers, M.S. Osofsky, B. Nadgorny, T. Ambrose, S.F. Cheng, P.R. Broussard, C.T. Tanaka, J. Nowak, J.S. Moodera, A. Barry, and J.M.D. Coey, Science 282, 85 (1998).

${ }^{5}$ For the scattering configuration we use the $a(b c) d$-type (Porto's) notation, where $a$ and $d$ are the directions and $b$ and $c$ are the polarization of the incident and scattered light, respectively.

${ }^{6}$ S.P.S. Porto, P.'A. Fleury, and T.C. Damen, Phys. Rev. 154, 522 (1967).

${ }^{7}$ D. N. Basov, E. J. Singley, C. P. Weber, A. Barry, and J. M. D. Coey (unpublished).
${ }^{8}$ I.I. Mazin, D.J. Singh, and Claudia Ambrosh-Draxl, Phys. Rev. B 59, 411 (1999).

${ }^{9}$ Peter Y. Yu and Manuel Cardona, Fundamentals of Semiconductors (Springer-Verlag, Berlin, 1996).

${ }^{10}$ G. Harbeke and E.F. Steigmeier, Solid State Commun. 6, 747 (1968); E.F. Steigmeier and G. Harbeke, Phys. Kondens. Mater. 12, 1 (1970).

${ }^{11}$ M. Iliev, G. Güntherodt, and H. Pink, Solid State Commun. 27, 863 (1978).

${ }^{12}$ In our low-temperature measurements we used an (110)- oriented film, where no scattering configurations for observation of the $B_{1 g}$ mode were available.

${ }^{13}$ M.N. Iliev, A.P. Litvinchuk, H.-G. Lee, C.L. Chen, L.M. Dezaneti, C.W. Chu, V.G. Ivanov, M.V. Abrashev, and V.N. Popov, Phys. Rev. B 59, 364 (1999). 